COUSIN, Olivier, L'effet établissement. Construction d'une problématique, Revue française de sociologie, 34:3 (1993:juil./sept.) p.395

R. franç. sociol. XXXIV, 1993, 395-419

\title{
Olivier COUSIN
}

\section{L'effet établissement}

\author{
Construction d'une problématique *
}

\begin{abstract}
RÉSUMÉ
La massification scolaire qui a d'abord touché les collèges, puis plus tardivement les lycées, a mis en avant l'hétérogénéité des établissements, qui a été principalement analysée par les sociologues en termes de performances. Si les établissements scolaires n'enregistrent pas des résultats identiques, cela ne tient pas uniquement à la nature de leur composition sociale mais aussi à leur organisation interne. A partir des différents travaux sociologiques portant sur l'établissement, nous distinguerons deux approches analytiques, l'une privilégiant les performances des élèves, l'autre s'attachant plus particulièrement à la politique des établissements. Nous essayerons alors de construire la problématique de «l'effet établissement», entendue comme la capacité des établissements scolaires à se construire comme unités pédagogiques et éducatives.
\end{abstract}

Depuis le début des années 80 , la sociologie de l'éducation a construit un nouvel objet communément appelé «l'effet établissement». Il s'agit d'observer la capacité des établissements scolaires à se construire comme des organisations autonomes. Cette problématique sous-entend que les établissements peuvent avoir leur propre efficacité, indépendamment des caractéristiques de leur public. Autrement dit, l'école n'est plus considérée comme un système monolithique agissant aveuglément de la même manière partout et au même moment, mais comme l'agrégation de multiples unités - écoles primaires, collèges et lycées - produisant chacune des effets sensiblement différents sur les résultats scolaires et/ou sur la sélection scolaire. Ainsi l'idée d'une diversification de la production scolaire en fonction de la nature de l'établissement voit-elle le jour. L'établissement est alors abordé comme une organisation qui possède un style particulier, des modes de relations sociales spécifiques et une capacité de mobilisation de ses acteurs et de ses ressources qui lui est propre. Il ne se réduit plus simplement aux contraintes qui pèsent sur lui. Les utilisateurs du système scolaire résument cette situation en proclamant que «toutes les écoles ne se

* Je tiens à remercier F. Dubet pour ses conseils et l'attention qu'il a bien voulu porter à ce travail. 
valent pas ». Ce glissement de problématique, de l'analyse du système à l'étude de l'unité de production, ne remet pas en cause l'orientation centrale de la sociologie de l'éducation, à savoir l'inégalité des chances devant l'enseignement. Il ne fait qu'affiner la relation globale observée entre origine sociale et réussite scolaire; face à des élèves différents, l'école n'apporte pas de réponse homogène.

\section{I. - Les grandes réformes depuis les années 60}

L'intérêt pour l'établissement est étroitement lié à l'histoire du système éducatif français, dont nous ne retracerons ici que les grandes lignes institutionnelles. Deux dates, deux réformes marquent les changements récents du système éducatif : 1963 avec la création d'un système cohérent, articulé autour de trois niveaux successifs, l'école primaire, le collège et le lycée ; 1975 avec la réforme des collèges qui uniformise les classe $\mathrm{s}$ de sixième et de cinquième et distingue définitivement l'école, le collège et le lycée. La réforme de 1963 marque l'abandon d'un système clos et peu perméable, où les filières sont fortement marquées socialement - primaire et son prolongement le primaire supérieur, secondaire qui seul conduit au baccalauréat. A l'issue de l'école primaire, tous les élèves entrent au collège, puis partent soit vers le second cycle long, sanctionné par le baccalauréat, soit vers le second cycle court afin de préparer un brevet d'études professionnelles. Cette réforme institue donc officiellement un système éducatif uniformisé. En réalité, à l'intérieur d'une même unité d'enseignement, essentiellement le collège, il existe des filières fortement hiérarchisées. La réforme de 1975, dite "réforme Haby », s'inscrit en partie dans la continuité des changements intervenus en 1963 et supprime les filières au collège. On parle alors véritablement de «collège unique» (1).

Une des conséquences de la création du collège unique est le développement de la fonction sélective du collège lui-même. En effet, auparavant, la sélection s'effectuait en grande partie en amont du collège, l'«élimination» était réalisée à l'extérieur du système scolaire. Le primaire donnait exceptionnellement la possibilité de poursuivre des études dans le secondaire. Accueillant principalement des enfants issus des milieux populaires, il conduisait vers des emplois précis à faible autonomie de décision, employés ou techniciens du commerce et de l'industrie. Le secondaire amenait les élèves vers le baccalauréat et trouvait son prolongement dans l'enseignement supérieur. Il accueillait essentiellement les enfants des couches

(1) Il ne s'agit ici que d'une présentation très partielle des réformes de 1963 et de 1975. Pour une histoire de l'enseignement et de ses grands changements, on se référera notamment à Prost (1986 et 1992); ainsi qu'à
Derouet (1992), plus particulièrement à la première partie pour la présentation des grands modèles de référence des acteurs du système scolaire. 
moyennes et supérieures à qui il n'offrait pas de qualification spécifique mais donnait accès aux emplois d'encadrement et de commandement (cf. Baudelot et Establet, 1971; Prost, 1992).

Avec l'unification du système éducatif et l'entrée de tous les élèves dans le secondaire, c'est au collège que vont se jouer l'orientation et la sélection, essentiellement après les classes de cinquième et de troisième. Les élèves les plus faibles, souvent les plus âgés, vont être orientés vers l'apprentissage - fin de $5^{\mathrm{e}}-$ ou vers le second cycle court - fin de $3^{\mathrm{e}}$. La bifurcation entre le cycle long et le cycle court, entre l'enseignement général et l'enseignement technique, se fait donc dorénavant au sein même du collège. La réforme des collèges fait reposer sur des critères scolaires la sélection qui antérieurement s'effectuait sur une base sociale. Elle a transformé en mérite ou en incapacité personnelle ce qu'on aurait auparavant imputé au hasard de la naissance. Le collège unique, que les enseignants réclamaient depuis de nombreuses années, va entraîner un profond mécontentement de la part du corps professoral et des syndicats (Robert, 1991). Le «malaise» des années 80-90 trouve en partie ses origines dans le changement introduit depuis les années 60.

Le collège unique signifie aussi et surtout l'accès de la plupart des élèves au deuxième cycle. Le système s'ouvre de fait au plus grand nombre. Ce qui ne va pas sans poser des problèmes tant la population nouvellement accueillie semble éloignée du modèle culturel dominant, c'est-à-dire de l'ensemble des valeurs et des objectifs visés par l'activité éducative. Le problème de l'hétérogénéité des élèves émerge et devient le cœur de la réflexion des enseignants. L'hétérogénéité se conjugue sous des formes diverses et ne se résume pas à la seule différence de niveaux entre les adolescents. Le collège unique est aussi confronté à une hétérogénéité de socialisation et d'aspiration chez les élèves. Le corps enseignant lui aussi se diversifie : dans les collèges les certifiés et les agrégés cèdent peu à peu le pas aux PEGC et aux maîtres auxiliaires. Ces «nouveaux» enseignants n'ont ni la même formation universitaire, ni le même statut administratif, ni la même charge de cours. Le système lui-même devient hétérogène avec la multiplicité des filières offertes aux élèves dans les lycées (2). Les établissements se distinguent et se hiérarchisent selon le type d'enseignement qu'ils proposent, classique, polyvalent ou technique. Une image du système éclaté et différencié se substitue à celle d'un système uniforme (3). Les réformes qui vont se succéder jusqu'au milieu des années 80 tenteront de résoudre le problème de l'hétérogénéité en renforçant l'autonomie des établissements.

(2) Il existe 60 baccalauréats différents, dont 28 pour le bac d'enseignement général et technique et 32 pour le bac professionnel, pour l'année 1991-1992.

(3) En réalité, le système éducatif français n'a jamais connu l'uniformité qu'on lui prête. J.-P. Briand et J.-M. Chapoulie (1992) montrent que, sous la Troisième République, les établissements se différenciaient selon les filières qu'ils proposaient et qu'il existait un «marché scolaire» variable selon les communes, par exemple. 
L'idée d'autonomie des établissements ne se réduit pas à la volonté politique de l'Education nationale, elle procède aussi d'une évolution des attentes des utilisateurs et des partenaires de l'école, parents. enfants, collectivités territoriales. L'image d'une institution, égalitaire el uniforme en tous points, disparaît au vu des résultats des établissements :égulièrement rapportés par la presse spécialisée et régionale. Les établissements sont classés et hiérarchisés et on parle alors de «bons» et de «mauvais » établissements. L'institution scolaire est soumise, elle aussi, à l'évaluation. La sectorisation vient renforcer l'impression d'une inégalité entre les différents établissements. Les parents désirent pouvoir choisir leur école et la «guerre scolaire» de 1984 en est l'illustration (Prost, 1986). Ils deviennent des «consommateurs d'école» et exigent de plus en plus souvent des comptes sur la production scolaire de l'établissement (Ballion, 1982 et 1992). Avec la décentralisation, les établissements se retrouvent sous la pression des familles et des instances de décision locales. Avec l'aval des instances ministérielles et rectorales, ils élaborent des projets parce que, selon $\mathrm{R}$. Ballion, «ils sont dans l'obligation de résultats». Le projet devient une véritable charte traduisant les objectifs à atteindre. Chaque établissement, en définissant son caractère propre, est amené à se singulariser. La logique n'est plus à l'uniformité mais à la différenciation. Le mouvement a débuté par les politiques reposant sur le volontariat des collèges en rénovation, il s'est officialisé ensuite avec l'obligation d'élaborer des projets d'établissement. Aujourd'hui, tous les types d'établissements scolaires sont concernés (Guillemet, 1992).

En devenant objet de politique et centre de décision, l'établissement devient objet de recherches alors qu'il était jusqu'alors perç $d$ uniquement comme une unité administrative permettant aux classes de fonctionner. La sociologie, dominée par la problématique de l'inégalité des chances devant l'enseignement, oscillait entre deux pôles, l'étude macro-sociologique de la relation entre l'origine sociale des élèves et leur chance de réussite scolaire d'une part, l'analyse micro-sociologique de la relation rnaîtres-élèves et des attentes des premiers vis-à-vis des seconds d'autre part. Le regroupement des recherches portant sur l'établissement ne corresfond pas pour autant à une unité d'analyse et encore moins à une Ecole. Bien que les supports théoriques et les problématiques soient variables, il est malgré tout envisageable de les regrouper sous deux rubriques générales. La première tendance réunit les recherches centrées autour de la notion d'efficacité, on parlera à ce propos de «l'école efficace». La deuxième tendance regroupe des études plus disparates mais qui ont en commun de s'attacher plus particulièrement à la capacité des établissements de connstruire leur «identité ». L'essentiel des recherches porte sur le collège, parce qu'il s'est imposé comme le carrefour du système scolaire et qu'il a été le premier confronté au problème de la massification. Il a aussi été le lieu d'expérimentation des réformes. 


\section{II. - Du système à «l'unité de production»}

Jusqu'au début des années 80 , la sociologie de l'éducation est marquée par la prédominance des «paradigmes hégémoniques». Quelles que soient les divergences théoriques des travaux essentiels, la sociologie est tournée vers la recherche des facteurs macro-sociaux de la réussite ou de l'échec scolaire. Les études sont centrées sur la relation entre l'origine sociale de l'élève et sa réussite scolaire (4). L'inégalité devant l'enseignement est comprise comme un effet de l'inégalité sociale (Baudelot et Establet, 1971). L'école est perçue comme une instance autonome de reproduction sociale, transmettant et inculquant les valeurs de la classe dominante et devenant un «agent» de celle-ci (Bourdieu et Passeron, 1964 et 1970). Pour d'autres, l'école n'a pas de fonction de sélection particulière, elle est essentiellement composée d'une succession de carrefours où les individus doivent effectuer des choix en fonction de leurs ressources et de leur ambition. L'inégalité doit alors être comprise comme le produit des combinaisons coût-risque-bénéfice qu'opère l'individu à chaque carrefour du système. Les calculs rationnels élaborés à chaque bifurcation ne se comprennent qu'en fonction de la position initiale de l'individu (Boudon, 1973).

Ces recherches présentent deux points en commun. D'une part, la variable «origine sociale» est placée au centre de tout le dispositif explicatif; d'autre part, les différences entre les établissements n'apparaissent pas. En France en particulier, l'école est vue comme un bloc uniforme servi par un corps enseignant, lui-même perçu comme un «agent » du système. Les résultats scolaires des élèves échappent en très grande partie à l'enseignant et à l'école en tant qu'organisation. Ce sont les mécanismes structurels ou l'agrégation des actions individuelles qui expliquent l'inégalité constatée. Entre le Ministère et l'élève il n'y a que des relais et, pour certains, rien.

Il faut dire que les enquêtes qui ont pris en considération les niveaux intermédiaires, notamment les variables scolaires, ont abouti à des résultats très décevants. Tel est le cas des travaux de J.S. Coleman et de son équipe qui concluent que les différences entre les établissements s'avèrent faibles (5). Le poids des différences entre les structures scolaires est presque toujours moins fort que celui des différences sociales lorsqu'il s'agit d'expliquer les inégalités de réussite scolaire. Cette étude conclut donc à la faiblesse de l'établissement et surtout à l'inefficacité des politiques visant à intervenir sur la structure scolaire. Seul un effet de composition émerge

(4) Pour une présentation exhaustive des travaux portant sur la sociologie de l'éducation, cf. Forquin (1982) et Van Haecht (1990).
(5) Cf. Coleman et al. (1966). Pour une présentation et une discussion des travaux de Coleman, on se référera à Cherkaoui (1979) et à Jencks (1979). 
de ces recherches. Coleman constate, en effet, que les minorités ethniques réussissent d'autant mieux en classe que la proportion d'élèves blancs est plus importante. Mais la politique des établissements elle-même n'est pas prise en compte, les établissements sont seulement situés sur un «marché ».

L'introduction de nouvelles références théoriques va permettre à la sociologie de l'éducation d'aborder les problèmes scolaires sous un angle différent. Le courant qui se rattache à «la nouvelle sociologie de l'éducation » en Grande-Bretagne marque un changement important parce qu'il se veut d'inspiration «anti-fonctionnaliste» et «anti-positiviste». L'image d'une société conçue comme un système composé d'éléments articulés, où l'individu est un produit social et l'éducation essentiellement un processus de socialisation, est remise en cause par une sociologie d'inspiration interactionniste. L'individu n'est plus un agent mais devient un acteur, et la société est appréhendée comme une scène où l'éducation apparaît comme un jeu de rôles ouvert (Forquin, 1983 et 1989). Ainsi, pour comprendre et analyser l'école, il faut regarder comment les acteurs la produisent, comment chacun définit son rôle et l'interprète. Cette orientation sociologique conduit les chercheurs à s'engager dans des études d'observation. Les histoires de vie, l'observation participante et les entretiens sont les méthodes couramment employées et préférées aux enquêtes statistiques. Malgré tout, l'établissement n'est pas le véritable centre d'intérêt des sociologues, le terrain reste un support méthodologique et l'orientation principale de ces recherches repose sur la construction des curricula. Bien qu'on ne puisse réduire à cet unique aspect théorique et méthodologique l'apport de la «nouvelle sociologie de l'éducation», il semble que la rupture entre les grandes enquêtes des années 60-70 et l'orienta:ion vers une sociologie des acteurs du système éducatif s'inspire en partie de ce courant de pensée.

L'émergence de la problématique de l'effet établissement provient aussi d'une réflexion critique et d'une relecture de grands travaux antérieurs. L'analyse secondaire des données disponibles, entreprise par certains, ne remet pas en cause les résultats généraux, notamment l'étroite relation entre l'origine sociale et la réussite scolaire. Mais la méthode utilisée pour traiter les informations est souvent affinée. Par exemple, M. Cherkaoui (1979) reprend les données fournies par le rapport Coleman et met particulièrement l'accent sur les variables contextuelles, en soulignant l'importance de l'effet de composition qui bénéficie aux minorités cthniques aux Etats-Unis et aux classes sociales défavorisées en général. Il montre aussi que les transformations de l'institution scolaire n'entraînent pas les mêmes conséquences pour tous. Ainsi, lorsque le système éducatif apparaît à ses utilisateurs comme transparent, avec des règles de fonctionnement claires et lisibles, les milieux défavorisés en tirent des bénéfices plus grands. L'introduction des variables contextuelles ouvre de nouvelles orientations qui peuvent rester dans le cadre de la recherche des facteurs d' əfficacité, ou prendre comme axe d'analyse les particularités du contexte à travers des études monographiques. 
Bien que les recherches portant sur l'établissement s'appuient sur des références théoriques diverses (6), il est possible de trouver un minimum d'unité intellectuelle dans cette approche de l'école. Il existe une volonté de rompre avec les analyses macro-sociologiques et de réfléchir sur la productivité de l'école à partir des spécificités locales. De même, ces études ne relient plus la réussite scolaire à la mobilité sociale.

\section{III. - L'école efficace}

1. La problématique de "l'école efficace» se caractérise par la recherche d'indicateurs permettant d'expliquer les facteurs de la "productivité» scolaire. Ces travaux se placent donc dans la lignée des grandes enquêtes relatives à la problématique de l'inégalité des chances, mais ils s'en démarquent nettement par leurs finalités. En effet, un de leurs objectifs est de rendre les indicateurs accessibles et utilisables par les acteurs du système scolaire. Les services statistiques du Ministère de l'éducation sont souvent partie prenante dans ce cadre de recherche et ils tentent de faire des établissements eux-mêmes des acteurs et des producteurs de recherches. La prise en compte de l'établissement est le deuxième facteur qui distingue cette problématique. Le contexte devient un élément central de ces études qui sont souvent des analyses comparatives entre des établissements. Enfin, elles sont généralement réalisées sur des échantillons de taille importante.

2. Les travaux de M. Duru et A. Mingat (1985, 1987, 1988 a et b) incarnent bien cette tendance. A partir d'une analyse contextuelle, ils montrent que les mécanismes d'orientation au collège peuvent varier entre les établissements. Pour ces auteurs, "les inégalités sociales ne peuvent se réduire au jeu de variables individuelles (valeurs scolaires, niveaux d'aspiration), mais résultent pour partie de différences de fonctionnement d'un contexte de scolarisation à l'autre». Par exemple, les procédures de passage dans la classe de $4^{\mathrm{e}}$ peuvent varier d'un établissement à l'autre et entraîner des différences importantes dans les taux de passage (7). Non seulement les établissements ne «produisent» pas la réussite ou l'échec de la même manière mais, de plus, ce ne sont pas les mêmes facteurs qui jouent selon les collèges. Ainsi, les variables individuelles et contextuelles retenues apparaissent plus ou moins discriminantes et leurs effets peuvent

(6) Deux autres axes théoriques ont aussi joué un rôle important dans le changement d'approche de l'école, l'ethnométhodologie et la sociologie des organisations.

(7) Les auteurs estiment que la différence peut être de l'ordre de $10 \%$, c'est-à-dire que, si la probabilité dans un collège moyen de passer en $4^{\mathrm{e}}$ est de $70 \%$ pour les élèves d'un profil scolaire et social donné, cette probabilité sera de $60 \%$ pour ces mêmes élèves dans un collège sélectif et de $80 \%$ dans un établissement plus généreux (Duru et Mingat, 1987). 
même s'opposer. C'est, par exemple, le cas de l'âge qui exerce au niveau individuel un effet négatif (plus l'élève est âgé, moins la probabilité de passer en $4^{\mathrm{e}}$ est grande), alors que son rôle devient légèrement positif au sein du collège (plus la population de l'établissement est âgáe, plus on a tendance à faire passer les élèves car il devient, en effet, difficile de les faire redoubler) (Duru et Mingat, 1988a). L'introduction de variables contextuelles ne remet pas en cause, bien évidemment, la relation entre origine sociale et réussite scolaire, mais elle permet de montrer que les mécanismes de sélection et d'orientation sont le résultat d'un jeu complexe de variables pouvant entraîner des conséquences non négligeables. Ainsi, soulignent les auteurs, ce sont essentiellement pour les élèves «moyens» que les établissements introduisent des différences, les décisions d'orientation étant quasi automatiques pour les autres.

Pour rendre compte de leurs observations, les chercheurs de I'IREDU ont recours à une modélisation mathématique de type probabiliste. Si ces modèles permettent d'estimer l'action de chacune des variajles dans les mécanismes d'orientation, ils ne permettent pas en revanche d'expliquer les différences constatées entre les collèges. Les auteurs soulignent les disparités entre les établissements et concluent à un effet de composition où tout se passe comme si le collège adaptait ses pratiques d'orientation à sa population dominante. Dans un établissement au recrutement social favorisé, les enfants des milieux populaires ont une probabilité plus forte de demander en premier choix la $4^{\mathrm{e}}$ et de l'obtenir que dans un collège plus populaire. Dans ce cas, il ne s'agit pas strictement d'un effet établissement, dans la mesure où les variations constatées entre les collèges échappent à la volonté des acteurs. Les auteurs constatent que les pratiques d'orientation sont plus sélectives dans les établissements populaires, mais ils ne peuvent l'expliquer à partir des variables retenues. Les recherches de l'IREDU servent de référence à la fois pour le modèle mis en place et pour leurs conclusions. Mais introduire les variables contextuelles ne peut suffire à comprendre les différences entre les établissements. I faut prendre en compte la notion d'établissement elle-même, autrement d t poser l'hypothèse générale que la politique des établissements, à travers la nature des relations sociales entre les acteurs et le projet pédagogique et éducatif mis en place, joue en partie sur les résultats scolaires des élèves.

3 . Les différentes recherches publiées régulièrement par la revue $E d u$ cation et formation présentent un autre aspect de la probématique de l'école efficace. Un des objectifs que se donnent les chercheurs de la DEP (8) est de construire des indicateurs les plus opératoires et accessibles possible afin de mesurer la production des établissements. L'évaluation est au cœur de ces recherches : que doit-on évaluer? comment définir la performance d'un collège? quels sont les indicateurs les plus opérants? Telles

(8) Direction de l'évaluation et de la prospective, Ministère de l'éducation nationale. On pourra se référer à la présentation

402 synthétique de D. Meuret (1986). Cf. également D. Trancart (1992). 
sont les questions que posent ces recherches empiriques. Les performances sont quasi exclusivement définies en termes quantitatifs et sur des critères de réussite scolaire. Mais ces études ont le mérite de ne pas se limiter aux taux de réussite aux examens. En suivant les élèves sur plusieurs années, les chercheurs de la DEP peuvent ainsi estimer, par exemple, des taux de «survie» par établissement. La réussite de l'élève n'est donc plus uniquement due à ses caractéristiques propres (âge, origine sociale), elle devient le résultat d'un processus qui trouve en partie son explication dans les composantes des établissements (9).

Ces études portent fréquemment sur des échantillons importants, mais l'unité de mesure reste l'établissement et son environnement. La comparaison de plusieurs établissements par l'analyse factorielle permet de mettre au jour les variables qui différencient les collèges selon deux principaux axes de discrimination indépendants l'un de l'autre: un axe rural/urbain - lié à l'orientation en fin de $3^{\mathrm{e}}$ - et un axe de structure sociale - lié à l'orientation en fin de $5^{\mathrm{e}}$ (Meuret, 1986). Sur chaque axe un ensemble de variables se regroupe, permettant d'opposer les établissements. Les variables concernent pour l'essentiel l'organisation interne du collège, elles sont, disent les auteurs, inter-corrélées. Sur le premier axe se trouvent par exemple, le niveau de qualification des enseignants, l'offre de langues vivantes, l'orientation en BEP et la proportion d'enfants d'agriculteurs. Le deuxième axe est constitué par les variables suivantes: les chances de «survie» à l'issue du cycle d'observation, la proportion d'enfants d'ouvriers, l'orientation en CAP et la proportion d'enseignants de type lycée. Ces recherches montrent une nouvelle fois que «le collège unique recouvre en réalité de grandes disparités » (Trancart, 1992). Mais la diversité ne se résume pas uniquement à la composition sociale des établissements. Elle tient en partie à l'organisation interne du système éducatif, c'est-à-dire aux décisions politiques ou administratives. Dans ce cas, en réalité, il ne s'agit pas de politique d'établissement, les décisions auxquelles font référence les travaux de la DEP émanent du Ministère ou des rectorats. Les collèges se caractérisent par le nombre d'enseignants de type lycée - certifiés ou agrégés -, le nombre d'élèves par classe ou encore le rattachement à une ZEP, ce qui ne dépend pas directement de la «volonté» de l'établissement.

Les analyses comparatives effectuées au sein de l'OCDE s'inscrivent dans la même logique, celle de la recherche d'indicateurs d'efficacité (Scheerens, 1989). Leur objectif est de tenter de les affiner le plus possible afin de pouvoir agir sur les établissements scolaires. Mais ce type de recherches bute rapidement sur une contradiction: plus on affine les indicateurs, moins ceux-ci ont de pouvoir explicatif et moins ils deviennent opérationnels. De plus, il est souvent très difficile de passer des concepts aux

(9) Nous faisons ici référence à l'étude dont les résultats ont été publiés dans la revue Education et formation de 1984 à 1986. 
indices, lorsque l'on cherche à quantifier ce qui est du domaine du subjectif. En effet, s'il semble aisé d'élaborer des indicateurs concernant les enseignants - ancienneté, niveau de diplôme, grade, taux de rotation ou encore taux d'encadrement - et de les rapporter à la performance d'un établissement, il devient beaucoup plus incertain de tenter d'evaluer l'effet des choix pédagogiques ou la nature des liens entre les enseignants sur les performances des élèves. Après avoir montré que tous les établissements ne produisent pas la même réussite, que le «contexte fait la différence », il faut se demander si la nature des relations sociale; à l'intérieur d'un établissement ne peut pas être aussi un moyen de rendre compte des disparités de performance.

4. Les travaux d'A. Grisay (1989) s'inscrivent dans le cadre de «l'école efficace». La recherche d'indicateurs est, là encore, au centre de l'étude. Mais la problématique s'élargit puisque, après avoir comparé des établissements ayant des caractéristiques communes et obtenant des résultats différents, l'auteur a pour objectif de rechercher l'explication de ces variations de performances dans le «fonctionnement de l'établissement»: «Des mêmes politiques peuvent produire des effets différents selon la nature de l'établissement et son recrutement social». Une typologie des collèges est constituée sur la base de deux variables dichotomiques, la performance des établissements et le recrutement social. Ainsi quatre types de collèges sont retenus, les favorisés/performants, les favo isés/non performants, les défavorisés/performants et les défavorisés/non performants (10). L'auteur compare les quatre types d'établissements en cherchant à mettre au jour ce qui les distingue, tant du point de vue de leur constitution que de la manière dont ils gèrent leur popu ation. Ce qui retient particulièrement l'attention, ce sont évidemment les cas atypiques, à savoir les établissements favorisés/non performants et les défavorisés/performants. C'est, en effet, ce type de collèges qui rompt le plus la relation entre origine sociale et réussite scolaire.

A. Grisay teste plusieurs hypothèses pour tenter d'expliquer les différences de performances entre les établissements: le rôle du contexte familial (suivi du travail, aide aux devoirs...), le contexte scolaire (taille et homogénéité sociale) et le climat de l'établissement. Ce dernier indicateur est particulièrement difficile à construire à cause de la complexité des items retenus (il s'agit pour l'essentiel de critères subjectifs) et de la présence éventuelle d'opinions contradictoires, le personnel de l'administration, les enseignants et les élèves n'ayant pas forcément les mêmes jugements. Ainsi, dans chaque type de collège, les indicateurs jouent do manière différente et c'est leur combinaison qui semble pouvoir expliquer les variations de performance. La notion de climat est intéressante parce qu'elle suppose que la vie de l'établissement est susceptible d'influencer les

(10) L'étude porte sur un échantillon de 60 collèges, les établissements «moyens » ayant été volontairement laissés de côté, précise A. Grisay. 
résultats des élèves. Il est donc possible de parler ici d'effet établissement même si la notion de climat est encore imprécise. Il ne s'agit pas encore d'action volontaire de la part des acteurs puisque celui-ci se résume aux relations «chaleureuses» ou non et à la perception d'un dynamisme. Le collège n'est pas encore tout à fait perçu comme un acteur social, autrement dit les objectifs qu'il se fixe ne sont pas connus, pas plus que les moyens mobilisés pour les réaliser, mais l'étude d'A. Grisay offre une synthèse et une ouverture intéressantes entre les recherches d'indicateurs d'efficacité et les travaux centrés autour des «styles d'établissement».

La construction de la typologie pose cependant quelques problèmes. En effet, la problématique de l' «effet établissement » n'est envisagée que pour les collèges «atypiques», c'est-à-dire lorsqu'un établissement, au vu de son recrutement social, ne répond pas à l'attente en termes de performance. A partir de la typologie d'A. Grisay - la construction de quatre «blocs» d'établissements - seule la comparaison «inter-blocs» est entreprise. Ce qui signifie que les établissements ne sont pas comparés les uns aux autres mais en fonction de la catégorie à laquelle ils appartiennent. Or il est envisageable d'aborder l'idée d'effet établissement non pas en cherchant les cas «atypiques » mais en retenant l'idée d'une variation de degré dans les performances. Car les collèges ne se départagent pas uniquement en «bons» ou «mauvais » établissements. La performance n'est pas une variable dichotomique mais continue. Ainsi, il ne faut pas se limiter aux établissements aux performances opposées, lorsqu'ils n'appartiennent pas au même type, mais il faut construire la comparaison par rapport à une échelle allant du meilleur au plus faible, ce qui permet de ne pas laisser de côté les établissements dits «moyens».

L'effet établissement ne peut être uniquement un renversement de tendance (rendre performant un établissement populaire, par exemple), il doit être perçu comme un processus qui entraîne des variations de degré. Ce qui signifie que deux établissements identiques en termes de recrutement social peuvent connaître des performances scolaires sensiblement différentes sans pour autant changer de catégorie. La performance, dans ce cas, est perçue comme graduelle et l'établissement le plus favorisé d'un échantillon n'est pas nécessairement le plus performant. Il semble donc utile de construire deux échelles: la première classe les collèges en fonction de leur recrutement social, il peut s'agir d'un indicateur simple (selon les CSP) ou d'un indicateur synthétique (combinaison des CSP et de l'âge moyen des élèves entrant en sixième par exemple) (11). La deuxième échelle répartit les collèges en fonction de leurs résultats. En comparant les deux échelles, plusieurs cas de figure se présentent que nous pouvons présenter sous forme de graphiques.

(11) L'introduction de l'âge n'est pas due au hasard compte tenu de la corrélation entre l'âge et la réussite scolaire. Cf. Duru et Mingat (1988 a). 
Revue française de sociologie
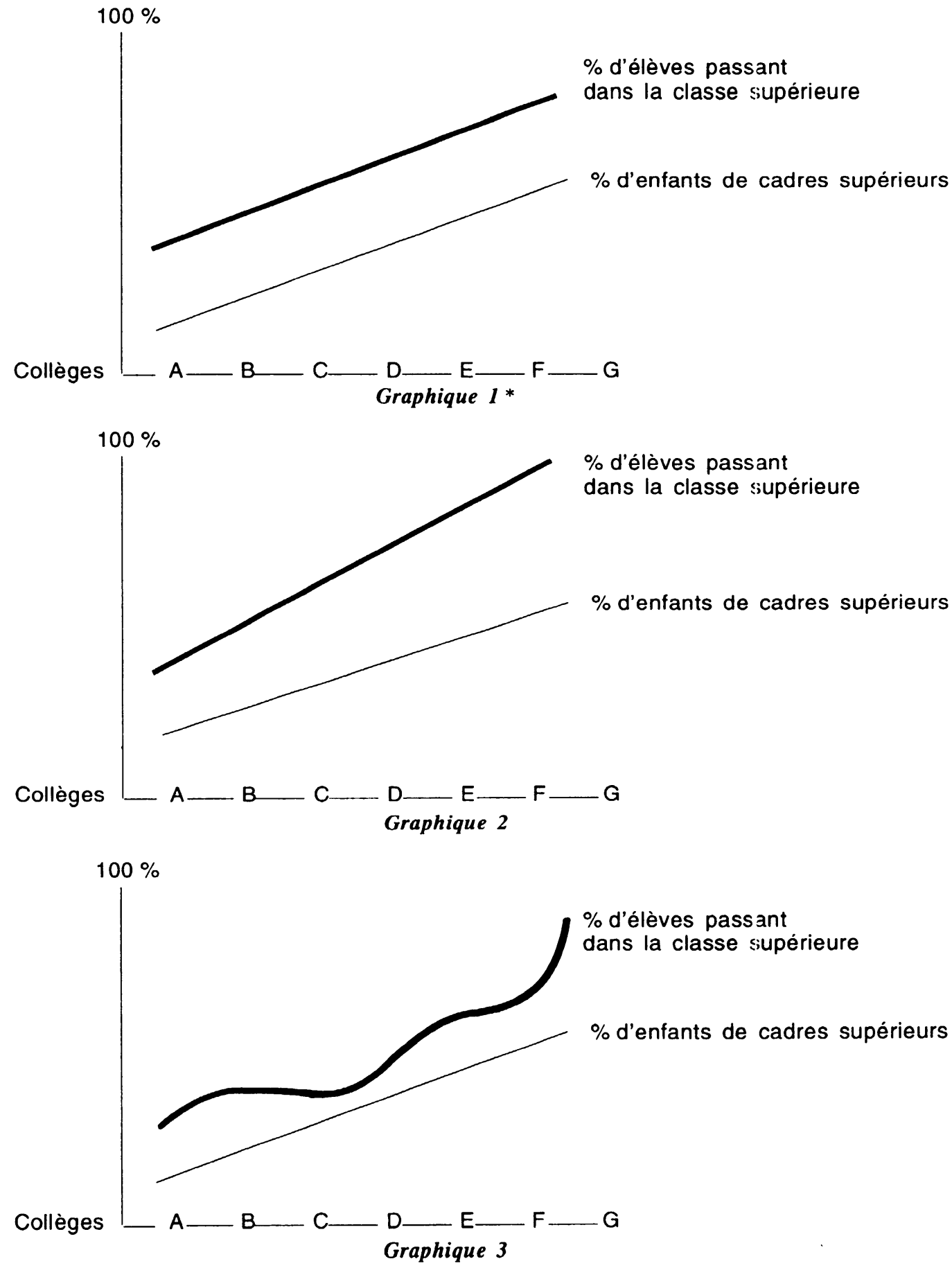

* Les graphiques se lisent de la manière suivante : la courbe maigre correspond au recrutement social des établissements, la courbe grasse aux résultats scolaires. Les points sur la droite des ordonnées correspondent aux établissements, leur ordre de classement est construit à partir de la courbe du recrutement social, allant des plus pıspulaires aux plus favorisés socialement.

406 
Dans le premier cas de figure, nous avons affaire à un effet linéaire de la composition du groupe sans interaction, tel que le définit J. Davis (12). Autrement dit, le succès d'un établissement dépend de sa composition sociale, mais les catégories sociales ont une réussite indépendante l'une de l'autre. Dans le cas présent, celle d'un établissement dépend uniquement de l'origine sociale des élèves.

Dans le deuxième cas de figure, il existe un effet de composition avec interaction entre les deux groupes de variables retenues (caractéristiques des élèves et performance). Les établissements favorisés réussissent mieux et, dans le même temps, plus un établissement accueille des enfants issus des catégories sociales supérieures, plus l'ensemble des élèves obtient de meilleurs résultats scolaires, quelle que soit leur origine sociale. Ce cas de figure théorique correspond aux observations du rapport Coleman pour les minorités ethniques qui réussissent mieux lorsqu'elles sont dans des écoles à majorité «blanche». Mais il ne s'agit pas non plus ici d'un effet établissement puisque la réussite scolaire des élèves dépend essentiellement de la composition sociale de l'établissement.

Le troisième cas de figure correspond à un effet établissement puisque la courbe des performances connaît des oscillations par rapport à la courbe du recrutement social. Les établissements les plus populaires enregistrent de faibles performances, mais certains obtiennent des résultats équivalents à des établissements plus favorisés. Ainsi l'ordre de classement des établissements en fonction du recrutement social ne correspond pas exactement à l'ordre de classement en fonction des résultats scolaires. Ce type de présentation offre l'avantage de ne pas s'intéresser uniquement aux cas «atypiques». La notion d'effet établissement est alors prise dans un sens moins restrictif et plus dynamique.

\section{IV. - L'établissement : une organisation sociale}

1. Nous regrouperons ici un autre ensemble d'études qui, lui aussi, ne constitue pas un courant de pensée uniforme. Mais ces recherches ont plusieurs points communs qui nous autorisent à les rassembler. D'une part, elles pensent l'établissement scolaire comme une organisation jouissant d'une relative autonomie. Cela sous-entend que les établissements ne peuvent être uniquement définis par les contraintes extérieures qui pèsent sur eux - recrutement social, zone d'implantation, etc. Au contraire, ils ont à leur disposition un certain nombre de ressources qu'ils sont capables de mobiliser dans le but de réaliser des objectifs. L'établissement est perçu comme un acteur social répondant à une situation qu'il analyse en fonction

(12) Nous nous référons ici à la typologie élaborée par J. Davis (1966). Le Graphique 1 correspond au type IIIa; le Graphique 2, au type IVa. 
des informations qu'il détient. La capacité de mobiliser les ressources est donc un des points importants de ces recherches.

D'autre part, l'efficacité de l'établissement n'est plus le point central de ces analyses. L'école, dans ce cadre, ne se résume pas à sa productivité scolaire, elle est aussi abordée comme une instance de socialisation remplissant un rôle éducatif. Rôle évidemment particulièrement difficile à évaluer. C'est pourquoi ces recherches font plus souvent appel à des méthodologies d'ordre qualitatif. L'accent est mis sur la nature des relations entre les acteurs sociaux, et une place importante est aıcordée à leurs discours.

2. D. Paty est à l'origine de l'un des tout premiers ouvrages en sociologie de l'éducation centrés autour de l'établissement (Paty, 1981). Cette étude monographique de douze collèges veut rompre avec l'approche dominante en ce domaine. La volonté de rupture est telle que le problème des performances et de l'évaluation n'entre pas dans le cadre de cette enquête. L'aspect éducatif de l'école en constitue l'enjeu principal à travers la constitution de son identité. Pour analyser le rôle éducat f d'un établissement scolaire, D. Paty puise ses références dans la socio ogie des organisations et s'attache plus particulièrement à étudier deux composantes d'une organisation : la structure, entendue comme l'ensemble des relations entre les divers groupes d'acteurs; le pouvoir, dans le sens de l'utilisation par les acteurs des ressources dont ils disposent.

D. Paty s'intéresse aux relations existantes entre style de relation sociale, style d'autorité du chef d'établissement et socialisation des élèves. Un tableau des différentes stratégies des élèves est dressé en fonction du type d'établissement dans lequel ils se trouvent scolarisés et des relations entre l'équipe d'administration et le corps enseignant. Pour l'auteur, les collèges se définissent en se référant soit à l'espace-classe, soit à l'établissement. Dans le premier cas, il est en réalité difficile ce parler d'une identité d'établissement tellement celle-ci est morcelée, puisque les classes sont indépendantes les unes des autres. L'identité d'un collège est aussi le résultat d'une action. Elle peut être, selon les cas, le fruit de la politique du chef d'établissement ou au contraire le résultat de l'engagement des enseignants.

Bien que D. Paty ne construise pas de réelle typologie des établissements autour de la fabrication de l'identité, cette approche modifie sensiblement le regard porté jusqu'alors sur les établissements scolaires. En effet, c'est la «boîte noire» qui est véritablement au cœur de l'analyse. Parce que les agents de l'éducation sont perçus comme de véritables acteurs sociaux, ils ne sont plus simplement définis par leur statut mais par leur action. Ainsi, le rôle des enseignants dépasse le cadre de la relation maître/élève, le professeur agit sur l'établissement et peut même devenir le héraut de son identité. La notion même d'identité est importante, puisque la différence entre les écoles ne se limite pas à leurs résultats. L'établissement existe au sens où il a la capacité de construire sa propre image et 
de la gérer, situation que les établissements ont parfaitement saisie et qui représente aujourd'hui un enjeu important puisque l'on parle de concurrence et de marché.

3. R. Ballion oriente ses recherches autour de la problématique du marché scolaire et des relations entre l'école et son public (13). La construction de l'identité d'un établissement scolaire est également le produit de l'image que s'en font les utilisateurs. L'application de la réforme Haby a eu notamment pour conséquence d'introduire des différenciations entre les établissements selon le type de public accueilli et les résultats obtenus. Au regard des familles, les établissements ne se valent pas et, depuis le début des années 80 , il existe une forte demande de choix, pour briser le système de la sectorisation. La liberté de choix, nous dit R. Ballion, «ce n'est pas, pour beaucoup de parents, l'expression de leur volonté d'optimiser les chances éducatives de l'élève en essayant d'obtenir un "meilleur établissement", c'est simplement le souhait de se protéger des risques, d'avoir une possibilité de recours s'il y a incompatibilité entre la demande éducative des familles et l'offre concrétisée par l'établissement» (1989). L'école n'est donc plus considérée comme un "service public au service de tous, mais comme étant au service du public devant répondre aux intérêts privés ». Malgré tout, si plus de $90 \%$ des familles sont favorables au choix de l'établissement, une très grande majorité $(85 \%)$ ne souhaite pas utiliser cette possibilité.

Cette nouvelle perception de l'institution scolaire n'a pas des répercussions sur la seule demande de scolarisation, elle participe aussi à la construction de l'image de l'établissement, constituant son identité extérieure. Bien que les acteurs de l'établissement vivent de manière cloisonnée, ils construisent une image interne relativement uniforme, précise R. Ballion. A l'inverse, l'image externe est le produit d'acteurs très divers - parents, presse, autres établissements... - où chaque partenaire puise ses informations à des sources particulières. L'image externe est souvent excessive, la rumeur peut prendre une place importante et, surtout, il n'existe pas de véritables indicateurs pour construire la figure de l'établissement. Les éléments retenus sont peu nombreux : les résultats aux examens, la composition sociale, l'architecture, la situation urbaine ou l'ancien statut de l'établissement pour les collèges. La variété des sources et la fragmentation de l'information ne sont pas équitablement partagées. Parce que la hiérarchie scolaire se confond avec la hiérarchie sociale, les catégories sociales les plus favorisées disposent d'un plus grand nombre de sources d'informations, qu'elles se procurent le plus souvent par elles-mêmes, elles représentent de «véritables parents d'élèves professionnels". Les familles modestes s'adressent, au contraire, plus souvent à ce que R. Ballion nomme les prédicateurs - conseillers d'orientation, enseignants... - et ces professionnels renforcent fréquemment la sélection et le poids de la

(13) Cf. Ballion (1982, 1986 et 1992) et Ballion et Euvrard (1989). 
reproduction. Elles sont «confirmées dans leur destin prévisible de classe, en l'occurrence choisir l'établissement le plus populaire, dans la mesure où le paradigme de la "reproduction" est le réfèrent dominant des agents à qui elles demandent conseil » (Ballion, 1989, p. 35).

Le passage d'un système unifié à un marché scolaire, où les parents deviennent des consommateurs, renforce les inégalités. Il est difficile de parler d'effet établissement dans ce cas mais les conséquences n'en sont pas moins importantes. Les établissements, à travers leurs orojets, sont en concurrence et, rappelle R. Ballion, ils sont dans l'obligation d'obtenir des résultats. Ils sont soumis à de fortes pressions extérieures et la recherche d'une couverture médiatique n'est pas rare, certains devenant même des «établissements vitrines». La définition d'une production de bien public devient floue et l'excellence apparaît comme le plus petit dénominateur commun. Les familles, qui ne possèdent qu'une information partielle, font et défont la réputation des établissements. Elles font, dit Ballion, du «darwinisme social» en choisissant ce qui est le plus choisi, prenant ce choix comme critère. Les «bons » établissements se retrouvent ainsi sur le devant de la scène alors que les établissements plus «faibles» peuvent devenir indésirables pour certaines familles. Le risque d'une ségrésation ethnique et sociale est réel.

4. L'hypothèse d'un effet établissement conduit aussi à nous interroger sur la capacité à construire l'identité des lycées et collègıs. Les travaux de J.-L. Derouet (1985, 1987, 1988 et 1992) s'organisent autour du principe de la constitution de l'identité sociale pour deux raiscns essentielles. D'une part, il est nécessaire de centrer l'analyse sur l'éta jlissement scolaire pour prendre en compte la diversité des situations. I a connaissance approfondie des processus de constitution de l'identité d'un établissement permet de connaître quels en sont les effets, dit-il. D'autre part, l'école ne propose plus de modèle «fort et intégré » distribuant ces rôles et des statuts. Il faut alors se tourner vers l'unité d'analyse qui offre encore une cohérence, à savoir l'établissement, parce que, selon Derouet, c'est au niveau de l'établissement que les acteurs peuvent redonner du sens à leur action (14).

Pour lui, l'établissement scolaire comprend une multitude de ressources latentes que le travail des individus connecte les unes aux autres pour constituer des réseaux. La situation n'est pas donnée en tant que telle, il faut la reconstruire en observant comment les acteurs interagissent. Ainsi, si les grandes enquêtes n'ont pu saisir l'importance de l'action de l'établissement, c'est parce que le croisement de variables est impuissant à

(14) L'«affaire du foulard» en est peutêtre l'illustration exemplaire. Le Ministère de l'éducation ne voulant et ne pouvant pas imposer un modèle, l'autorisation ou non pour les filles de porter dans les établissements et/ou en classe le «foulard islamique » a lais- sé aux établissements le choix de décider s'ils acceptaient ou non ces élèves. Le Ministère s'est contenté de rappeler que le prosélytisme est interdit dan: les établissements scolaires. 
rendre compte des effets cumulatifs qui font l'effet établissement. Mais, surtout, leur principe «est de faire ressortir les ressemblances entre les phénomènes sociaux, il est alors parfaitement illusoire de leur demander de mettre en relief des différences ».

Depuis les critiques remettant en cause le principe de l'égalité des chances, l'Etat serait dans l'incapacité de proposer un modèle de «justice». Affaibli depuis le début des années 80 , il ne peut mettre en place des normes en dehors des programmes. Il revient donc au niveau local, écrit J.-L. Derouet, de trouver des accords sur les objectifs à atteindre. L'établissement devient donc le lieu où les acteurs tentent de «s'accorder» sur une définition du bien commun. L'auteur distingue trois types de principe de justice : la compétence civique qui renvoie à l'universalisme, c'est-àdire à la priorité donnée à la connaissance et à un enseignement standard, loin de tout particularisme; la compétence domestique qui, au contraire, s'appuie sur l'épanouissement de l'élève dans son milieu; la compétence industrielle qui vise l'efficacité en favorisant la sélection dans un but de progrès collectif, l'enseignement se définissant alors par objectifs. Ces trois compétences représentent des idéal-types, et le chercheur doit comprendre comment elles s'articulent. Existe-t-il un accord sur les objectifs ? Telle est la question posée.

L'approche de l'établissement en termes d'objectifs que se fixent les acteurs et de références qui les inspirent pour y parvenir renvoie une image dynamique de l'institution scolaire. Mais il devient alors nécessaire de se demander quelles sont les conséquences de ces accords et surtout quels en sont les effets. Il est probable que les logiques d'action ne produisent pas les mêmes effets. Il est aussi envisageable que les effets ne soient pas identiquement partagés par tous les élèves. Il paraît difficile de se limiter à une analyse en termes d'identification des objectifs. Dans ce cas, il ne peut s'agir d'effet établissement.

Il faut aussi s'interroger sur la capacité de passer d'une référence centrée sur la classe à une norme d'établissement. Car identifier les principes des compétences auxquelles se réfèrent les acteurs ne nous dit pas quels sont les objectifs poursuivis par l'établissement. L'établissement scolaire est aussi un lieu de pouvoir où s'exercent des contraintes qu'il convient d'introduire dans la définition de la capacité des acteurs à agir. S'il est vrai qu'une autonomie d'action et de décision est formellement reconnue aux établissements, il est aussi vrai qu'il existe une culture enseignante qui ne va pas forcément dans le sens de la participation. Les professeurs se réfèrent d'abord à leur matière, puis à leurs classes et beaucoup plus rarement à un établissement. Lorsque l'on parle de politique d'établissement, il faut bien voir quelles en sont les limites. En effet, la participation ne concerne le plus souvent qu'un petit nombre d'acteurs, et il n'est pas rare que les objectifs affichés ne soient portés que par le chef d'établissement. Les résistances au sein des établissements restent réelles bien que la norme soit à l'élaboration de projets qui eux-mêmes apparaissent inconsistants et 
proposent rarement une action d'ensemble concernant tout l'établissement (15).

5. L'étude comparative de trois collèges populaires de la banlieue d'une grande ville de province réalisée par F. Dubet, O. Cousin et J.-P. Guillemet (1989) répond en partie à ces interrogations. L'objectif de cette recherche était double : pourquoi les acteurs d'un établissement scoiaire se mobilisent-ils et quelles sont les conséquences de cette mobilisation sur les performances des élèves? Par mobilisation, il faut comprendre la volonté des acteurs de construire une «norme » d'établissement, c'est-à-dire de ne plus simplement se définir par rapport à des classes ou à des heures de cours indépendantes les unes des autres. L'enjeu n'est pas tant cle mesurer l'efficacité des collèges que de tenter de vérifier l'existence d'un lien entre, d'un côté, la participation des acteurs à la vie de l'établissement et, de l'autre, la réduction des inégalités sociales et scolaires (16). C'est pourquoi trois collèges au recrutement social très proche ont été retenus. L'évaluation de la performance des élèves se limite aux résultats obtenus au brevet des collèges et au passage en second cycle long. La sélection est mesurée selon deux dimensions, scolaire - sur l'ensemble de la population des élèves d'un collège - et sociale - selon le milieu d'origine des collégiens.

Il paraît possible de relier la mobilisation des acteurs aux performances de l'établissement. Ainsi, le collège qui connaît la plus forte participation de ses acteurs autour des objectifs préalablement définis est aussi celui qui s'avère le moins sélectif tant scolairement que socialement. Certes, ses performances restent médiocres (17), mais il semble que dans ce collège la sélection en fin de $5^{\mathrm{e}}$ et de $3^{\mathrm{e}}$ soit très atténuée et surtout qu'elle soit la plus égalitaire. Au contraire, dans le collège qui ne connaît aucune cohésion, la sélection sociale joue librement dans l'univers atomisé de l'établissement, les enfants des classes populaires se trouvant être les plus pénalisés (18). Il convient donc de s'interroger sur les facteurs de la mobilisation. Le collège «mobilisé » est celui qui cherche volontairement à surmonter l'hétérogénéité des classes et des méthodes pour se construire

(15) Cf. «Les collèges en panne», Le Monde du 2 avril 1992.

(16) Dans le cadre d'une comparaison entre des établissements scolaires, il semble pertinent de distinguer la sélection sociale de la sélection scolaire. La première, la plus couramment employée, ne prend en compte que la variable «origine sociale » comme élément de comparaison. La deuxième compare les établissements à chaque palier de l'orientation sans distinguer les élèves. Ainsi, la sélection peut affecter tous les élèves (elle est alors essentiellement scolaire), ou seulement une catégorie d'entre eux (selon l'origine sociale), ou encore combinis les deux.

(17) En effet, $50 \%$ se dlement des enfants entrés en $6^{\mathrm{e}}$ obtiennent le brevet des collèges quatre années plus tard.

(18) Pour le premier collège, $38 \%$ des élèves de $6^{\mathrm{e}}$ entrent en seconde quatre années plus tard; $25 \%$ des enfa its des milieux populaires et $35 \%$ des enfants des classes moyennes obtiennent le brevet en quatre ans. Pour le deuxième établissement cité, les données sont respectivement de $30 \%, 14 \%$ et $45 \%$ (Dubet et al., 1989 1. 
comme établissement. Une «norme» d'établissement s'esquisse, qui peut prendre des aspects différents et résulte de plusieurs facteurs.

Deux types d'acteurs sociaux sont à la source de cette capacité de mobilisation : l'équipe de direction et les enseignants. Leur rôle doit être analysé comme le produit d'une interaction entre les différentes définitions que chacun donne de son action et de sa tâche, en fonction de la nature de l'établissement et de son environnement. La pression exercée par les familles et par le «marché scolaire»n'est évidemment pas négligeable. La mobilisation permet essentiellement de briser l'isolement des professeurs et offre une cohérence dans l'activité pédagogique et éducative de l'établissement. Elle rend compte d'un effet établissement qui mesure moins ici les performances pures d'un collège que la possibilité d'intervenir, d'atténuer ou de renforcer les «déterminismes» socio-culturels.

Dans la continuité de cette recherche sur les collèges, une étude comparant six lycées met en avant des phénomènes comparables (19). Dans ce cas, il s'agissait d'expliquer pourquoi des établissements scolaires connaissent des variations de performances dans le temps. Afin de tester l'hypothèse d'un effet établissement - ce qui suppose que la politique menée par un lycée soit susceptible de rendre compte, en partie, de ses performances -, les chercheurs se sont assurés que les lycées étudiés n'avaient pas connu de changements structurels notables. Ainsi, sur les trois années retenues, ni le recrutement social des lycées ni l'offre de sections et de filières n'ont été modifiées. La performance des établissements est définie selon deux indicateurs qui privilégient la capacité des lycées à amener le maximum d'élèves jusqu'au baccalauréat (20). Le premier indicateur correspond au nombre d'élèves ayant 15 ans en seconde et qui obtiennent le bac en trois ans - indicateur longitudinal -, le deuxième consiste à rapporter le nombre de bacheliers au nombre d'élèves sortant de l'établissement - indicateur transversal. Sur les trois années étudiées, les lycées vont connaître une «hausse» ou une «baisse» de leur performance.

Il semble, a priori, que la variation des performances des lycées soit étroitement liée à la massification des effectifs. En effet, tous les lycées «en baisse» ont accusé une augmentation de leur effectif presque deux fois plus importante que les lycées «en hausse». En réalité, la relation entre massification et performances des établissements est plus complexe et doit être nuancée. La corrélation existe au niveau de l'établissement mais elle n'est plus toujours vérifiée quand on analyse les performances par niveaux d'études et par sections. Autrement dit, il existe dans les lycées en hausse des sections qui ont connu un fort taux d'accroissement de leurs effectifs sans pour autant enregistrer des baisses de leurs performances et,

(19) Cf. Cousin et Guillemet (1992). Parmi les six lycées retenus pour cette étude, cinq accueillent principalement des élèves d'origine sociale modeste et populaire.
(20) Ces indicateurs ont été élaborés par les services statistiques de la Direction de l'évaluation et de la prospective (cf. DEP, 1990). 
Revue française de sociologie

inversement, un faible accroissement des effectifs ne signifie pas automatiquement une «hausse» des performances. Ainsi, on peut faire l'hypothèse que la variation des performances est moins due à la massification qu'à une capacité des établissements de la prendre en charge par une politique volontariste. Il s'avère donc possible de rechercher les causes de ces variations des performances dans les caractéristiques de l'établissement. Par caractéristiques, il faut comprendre le style de la directicn, le degré de mobilisation des enseignants, la nature des choix et des initiatives pédagogiques et, enfin, la politique de gestion des flux des élèves. La variation des performances des lycées est le produit de plusieurs facteurs qui cumulent leurs effets.

Comme les collèges, les lycées mobilisés réussissent à mettre en place des actions pédagogiques afin de répondre à la massification alors que, dans les établissements «en baisse », l'absence de cohésion ne permet pas de gérer ce nouveau public. Les établissements «en hausse» présentent une combinaison de facteurs par bien des points opposée à celle des établissements «en baisse» : équipe de direction au style «laisser-faire», enseignants n'entretenant pas de rapports conflictuels, professeurs partageant une expérience commune, valorisation des élèves et refus de parler de «baisse de niveau», d'une part; équipe de direction au style «administratif», relations conflictuelles entre les enseignants, refus du travail en commun, vision passéiste et négative des élèves pour les lycées en baisse, d'autre part (21).

Sans aller jusqu'à affirmer qu'il existe une relation causale entre la mobilisation des acteurs et la variation des performances des établissements, on constate malgré tout un lien étroit entre ces deux phénomènes. La mobilisation des acteurs, construite dans le cadre de ces études de manière empirique, regroupe plusieurs dimensions: une redéfinition du rôle de l'équipe de direction qui souhaite sortir d'une action orientée autour des tâches administratives pour s'impliquer plus ouvertement dans l'action pédagogique; une redéfinition du champ d'intervention des enseignants vers une action collective par le biais d'une concertation soit disciplinaire soit interdisciplinaire; une adhésion à des objectifs communs partagés par le plus grand nombre, qui permet de construire une norme collective. La mobilisation peut être éclatée ou collective. Dans le premier cas, il s'agit de micro-initiatives couvrant le champ éducatif ou le champ pédagogique, qui concernent soit un seul noyau de professeurs soit plusieurs petits groupes. Dans le deuxième, les enseignants travaillent dans une même orientation plus clairement définie par les acteurs. Le chef d'établissement s'apparente

(21) Le style de direction «laisser-faire» renvoie à une équipe d'encadrement qui laisse les enseignants libres d'agir. Elle peut, selon les cas, se contenter de gérer l'administratif et d'annoncer des consignes de travail sans vraiment s'investir de près ou de loin dans la vie de l'établissement. La direction «administrative » définit son rôle de manière étroite, exclusivemt:nt centré sur les tâches et les fonctions adn inistratives, et elle freine souvent les initiatives. 
alors à un «chef d'orchestre». Le degré de mobilisation a des incidences sur les résultats des établissements scolaires puisque que ce sont les plus mobilisés qui enregistrent les performances les plus élevées (22).

Etablir un lien entre une politique et les résultats d'un établissement laisse en suspens la question des facteurs de la mobilisation. Pourquoi les acteurs passent-ils d'une définition étroite de leur rôle à une définition plus collective? Ou, pour le dire autrement, comment passe-t-on de la référence classe à une norme établissement? Il ne semble pas que nous puissions nous appuyer sur les théories classiques de la mobilisation collective dans le cas de l'école (23). Nous ne pouvons, dans l'état actuel des connaissances, que dresser un catalogue des éléments qui apparaissent être des facteurs de la mobilisation des acteurs. L'équipe de direction, tant dans les lycées que dans les collèges, joue un rôle non négligeable puisqu'elle peut être à l'origine d'un changement des pratiques. Elle peut par exemple offrir aux enseignants des heures de concertation et briser ainsi leur isolement, elle peut faciliter les stages pédagogiques pour les professeurs ou, plus simplement, proposer un bilan détaillé de la production scolaire de l'établissement, créant de ce fait une prise de conscience collective. Mais la bonne volonté d'une équipe de direction ne peut suffire. Elle doit pour ce faire trouver un «terrain favorable», être relayée par des enseignants prêts à travailler «autrement». Cette mobilisation trouve ses racines aussi bien dans un engagement politique ou syndical des enseignants, dans la pression que peut exercer l'environnement, dans le désir de renverser l'image de l'établissement ou du métier d'enseignant, ou encore dans le hasard des réseaux d'amitié qui se constituent et qui peuvent donner l'envie de travailler ensemble.

Les facteurs de la mobilisation restent donc encore à définir et à comprendre (24), les recherches sur l'effet établissement ne peuvent pas se limiter à la seule mesure de leurs conséquences. Pour étudier les facteurs favorables à la mobilisation des acteurs, il est nécessaire de rechercher comment ceux-ci définissent leur situation. La mobilisation n'est pas une donnée en soi, elle résulte de la façon dont l'individu perçoit son environnement et de la situation à laquelle il doit faire face, qui dépend donc à la fois de l'ordre social tel qu'il se présente à lui et de son histoire personnelle à travers ses engagements politiques, moraux et professionnels (25). Il paraît donc nécessaire de dissocier la réalité telle qu'elle peut

(22) C'est le cas notamment des lycées "en hausse»: celui des trois lycées de cette catégorie qui voit ses performances s'améliorer le plus nettement est l'établissement qui connaît une mobilisation forte et collective (cf. Cousin et Guillemet, 1992).

(23) Pour une présentation critique des travaux classiques et récents portant sur les théories de la mobilisation collective, cf.
Mann (1991).

(24) La difficulté s'accroît lorsqu'on prend en compte le facteur temporel. Ainsi, les politiques et la mobilisation s'avèrent extrêmement fragiles dans le temps (cf. Cou$\sin , 1992)$.

(25) Nous nous référons ici à l'approche sociologique de l'Ecole de Chicago. Cf. Grafmeyer et Joseph (1979) et Coulon (1992). 
être perçue et vécue par les acteurs de la réalité «objective». Ainsi les recherches qui partent de l'analyse des performances scolaires doivent-elles s'interroger sur la définition que donnent les acteurs de la réussite des établissements.

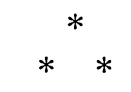

A la lecture de ces différentes recherches, il est possible de cerner ce que recouvre la notion d' «effet établissement». Cette proslématique mesure en réalité trois phénomènes complémentaires : la sélection scolaire, la sélection sociale et la socialisation. Comme l'efficacité est le plus souvent évaluée par rapport à la performance scolaire, les recherches tentent d'apprécier la réussite des élèves selon la nature des établis sements. L'évaluation s'effectue à chaque palier de la scolarité. Mais l'efficacité d'un établissement peut aussi se mesurer socialement, à partir des catégories sociales qui réussissent le mieux. Dans ce cas, les chercheurs tentent d'estimer la capacité des établissements à réduire les inégalités sociales. Or, un établissement scolairement efficace ne l'est pas toujours socialement, et inversement. Il est donc nécessaire de prendre en compte les deux aspects de l'efficacité pour apprécier les conséquences de la politique des établissements.

Mais l'efficacité n'est pas un concept univoque tant pour les acteurs du système scolaire que pour les sociologues. Il convient donc de s'accorder sur l'unité de mesure. Il semble à ce propos que l'efficacité comprise comme la capacité d'amener le maximum d'élèves au plus haut niveau possible soit la définition la plus riche. Le suivi de cohortes permet d'évaluer plus précisément la «productivité» des établissements.

Le troisième aspect de l'effet établissement concerne la socialisation des élèves. En effet, l'évaluation de la mobilisation des acteurs ne se réduit pas à l'efficacité de l'établissement : d'une part, elle a des conséquences sur l'organisation sociale de la vie des élèves; d'autre part, l'efficacité d'une politique n'entraîne pas nécessairement l'adhésion des élèves. Ces derniers peuvent percevoir la mobilisation des acteurs cornme un renforcement du contrôle social à leur égard ou, plus prosaïquement, comme une surcharge de travail (26). Mesurer l'effet établissement revient donc à prendre en compte ces trois dimensions et à regarder comment elles s'articulent.

(26) C'est le cas pour un des collèges étudiés par F. Dubet et al. (1989). Le collège le plus mobilisé et le plus efficace est perçu par les élèves comme trop contraignant parce qu'ils sont constamment sollicités par les enseignants et l'administration. 
La prise en compte de la socialisation des élèves nous oblige aussi à les considérer non plus comme des agents du système scolaire mais comme des acteurs. Il convient donc de s'interroger sur l'expérience des élèves et de les considérer comme des acteurs construisant leur expérience à travers des choix culturels et des orientations. Les élèves élaborent des stratégies et les significations de leurs stratégies dans un système de relations sociales. F. Dubet (1991) a montré que les expériences des lycéens variaient en fonction de la nature du lycée (lycée classique, polyvalent ou LP). Il est possible de reprendre cette problématique en la reliant à la capacité de construction de l'établissement.

Sans faire de la problématique de l'effet établissement un facteur d'explication hégémonique de la production scolaire, il est souhaitable d'élargir les recherches à l'ensemble de l'établissement, c'est-à-dire de percevoir les écoles comme des organisations productrices de biens sociaux, culturels et pédagogiques (27). L'hypothèse d'un effet établissement permet de ne pas réduire celui-ci à un producteur de réussite ou d'échec scolaire. Au contraire, il apparaît comme une organisation sociale complexe qui répond à des intérêts divers et contradictoires (le marché et les usagers) et qui, à l'aide de ressources humaines, matérielles et symboliques, doit résoudre un certain nombre de problèmes par l'action collective. L'établissement n'existe donc que lorsque les acteurs ont la capacité de passer d'une organisation fragmentée à une organisation d'ensemble.

Olivier COUSIN

CADIS, EHESS-Université de Bordeaux II Domaine universitaire, 33400 Talence

(27) Ces trois dimensions de la production scolaire sont de plus en plus prises en compte dans les interrogations sur l'école, mais elles ne sont pas nécessairement reliées, comme le montrent les différentes recherches portant sur les problèmes du retour de l'autorité ou de la citoyenneté liés à l'immigration, par exemple.

\section{RÉFÉRENCES BIBLIOGRAPHIQUES}

Ballion R., 1982. - Les consommateurs d'école, Paris, Stock.

— 1986. - «Le choix du collège : le comportement 'éclairé' des familles», Revue française de sociologie, 27 (4), pp. 719-734.

- 1992. - La bonne école, Paris, Hatier.

Ballion R., Euvrard F., 1989. - Le choix du lycée, Paris, Ministère de l'Education Nationale, DEP.

Baudelot C., Establet R., 1971. - L'école capitaliste en France, Paris, Maspero.

Boudon R., 1973. - L'inégalité des chances, Paris, Armand Colin.

Bourdieu P., Passeron J.-C., 1964. - Les héritiers, Paris, Editions de Minuit.

- 1970. - La reproduction, Paris, Editions de Minuit. 
Revue française de sociologie

Briand J.-P., Chapoulie J.-M., 1992. - Les collèges du peuple, Paris, Fditions du CNRS.

Cherkaoui M., 1979. - Les paradoxes de la réussite scolaire, Paris, Presses Universitaires de France.

- 1986. - Sociologie de l'éducation, Paris, Presses Universitaires de France.

Coleman J.S. et al., 1966. - Equality of educational opportunity, Washington, Department of Health, Education and Welfare.

Coulon A., 1992. - L'Ecole de Chicago, Paris, Presses Universitaires de France.

Cousin O., 1992. - «Mobilisation au collège», dans «L'école à vocaticns multiples », Informations sociales, $\mathrm{n}^{\mathrm{o}} 18$.

Cousin O., Guillemet J.-P., 1992. - «Variations des performances scolaires et effet d'établissement », Education et formation, $\mathrm{n}^{\circ} 31$, pp. 23-30.

Davis J., 1966. - «Les effets de la composition d'un groupe : technique d'analyse», dans R. Boudon et P. Lazarsfeld (eds), L'analyse empirique de la causali.é, Paris, La Haye, Mouton, tome II, pp. 170-180.

DEP, 1990. - Une fiche d'indicateurs pour les lycées sur les résultats au liaccalauréat, Paris, juillet.

Derouet J.-L., 1985. - «Des enseignants sociologues dans les établisse nents ? Ethnologie de terrain et contrôle sociologique dans l'étude du fonctionnement des śtablissements scolaires", Revue française de pédagogie, $\mathrm{n}^{\mathrm{0}} 72$.

- 1987. - «Une sociologie des établissements scolaires», Revue françıise de pédagogie, $\mathrm{n}^{\mathrm{0}} 78$.

- 1988. - Désaccords et arrangements dans les collèges. 1981-1986, Paris, Institut National de la Recherche Pédagogique.

- 1992. - Ecole et justice, Paris, Métailié.

Dubet F., 1991. - Les lycéens, Paris, Le Seuil.

Dubet F., Cousin O., Guillemet J.-P., 1989. - «Mobilisation des établi`sements et performances scolaires. Le cas des collèges », Revue française de sociologie, ¿0 (2), pp. 235-256.

Duru M., Mingat A., 1985. - De l'orientation de fin de cinquième au fonctionnement du collège, tome 1 : Evaluation de la procédure, Dijon, Cahiers de l'IRE:Ju, $\mathrm{n}^{\circ} 42$.

- 1987. - «Facteurs institutionnels de la diversité des carrières scolaires », Revue française de sociologie, 28 (1), pp. 3-16.

- 1988a. - De l'orientation en fin de cinquième au fonctionnement dl collège, tome 2 : Progression, notation, orientation : l'impact du contexte de la scolar sation, Dijon, Cahiers de l'IREDU, $\mathrm{n}^{\circ} 45$.

- 1988b. - «Le déroulement de la scolarité au collège : le contexte 'fait des différences'...», Revue française de sociologie, 29 (4), pp. 649-666.

Forquin J.-C., 1982. - «L'approche sociologique de la réussite et de l'éıhec scolaire : inégalités de réussite scolaire et appartenance sociale ", Revue française d' pédagogie, no ${ }^{\circ} 59$ 60.

- 1983. - «La 'nouvelle sociologie de l'éducation' en Grande-Bretagne : orientations, apports théoriques, évolution (1970-1980)», Revue française de pédago :ie, $\mathrm{n}^{\circ} 65$.

- 1989. - Ecole et Culture. Le point de vue des sociologues britanniques, Bruxelles, Editions de Boeck.

Grafmeyer Y., Joseph I., 1979. - «Présentation» de L'Ecole de Chicıgo, Paris, Champ Urbain.

Grisay A., 1989. - Quels indicateurs d'efficacité pour les établissement:s scolaires? Etude d'un groupe contrasté de collèges "performants» et "peu performants", Université de Liège, Service de Pédagogie Expérimentale.

Guillemet J.-P., 1992. - «L'école au pluriel », dans «L'école à vocations multiples », Informations sociales, $\mathrm{n}^{\mathrm{o}} 18$. 


\section{Olivier Cousin}

Jencks C., 1979. - L'inégalité, influence de la famille et de l'école en Amérique, Paris, Presses Universitaires de France.

Mann P., 1991. - L'action collective, Paris, Armand Colin.

Meuret D., 1986. - «Les disparités entre collèges publics. Analyse d'ensemble », Education et formation, $\mathrm{n}^{\mathrm{0}}$ 8, pp. 11-33.

Paty D., 1981. - Douze collèges en France, Paris, La Documentation Française.

Prost A., 1986. - L'enseignement s'est-il démocratisé?, Paris, Presses Universitaires de France.

- 1992. - Education, société et politiques, Paris, Le Seuil. Sociologie de l'éducation. Dix ans de recherche, recueil de notes de synthèse publiées par la Revue française de pédagogie, Paris, InRP, L'Harmattan, 1990.

Robert A., 1991. - «Syndicalisme enseignant et lutte contre l'échec scolaire», Revue française de pédagogie, $\mathrm{n}^{\circ}$ 95, pp. 5-19.

Scheerens J., 1989. - Indicateurs internationaux de l'enseignement, Paris, Centre pour la Recherche et l'Innovation de l'Enseignement.

Trancart D., 1992. - «Disparités entre collèges publics», Education et formation, n ${ }^{\circ} 31$, pp. 11-21.

Van Haecht A., 1990. - L'école à l'épreuve de la sociologie. Questions à la sociologie de l'éducation, Bruxelles, Editions Universitaires, Editions de Boeck. 